\title{
Pitfalls in Diagnosing a Tension Pneumopericardium-A Case Report
}

\author{
Shankar Hanamantrao Hippargi ${ }^{1}$, Vinayak Tonne ${ }^{2}$ \\ ${ }^{1}$ Department of Accident \& Emergency Medicine, Meenakshi Mission Hospital \& Research Center, Madurai, India; ${ }^{2}$ Department of \\ Imaging Science, Medwin Multispecialty Hospital, Hyderabad, India. \\ Email: drshank18@gmail.com
}

Received January $17^{\text {th }}, 2013$; revised March $5^{\text {th }}, 2013$; accepted April $15^{\text {th }}, 2013$

Copyright (C) 2013 Shankar Hanamantrao Hippargi, Vinayak Tonne. This is an open access article distributed under the Creative Commons Attribution License, which permits unrestricted use, distribution, and reproduction in any medium, provided the original work is properly cited.

\begin{abstract}
A 65-year-old female patient was brought to our emergency department (ED) with alleged history of road traffic collision (RTC). The patient had respiratory distress on arrival and hence she was immediately intubated and ventilated. Blood pressure and peripheral pulses were not measurable; however the central pulses were present. Aggressive fluid resuscitation was started. Primary assessment revealed distended neck veins, bony crepitus over right chest. Bedside plain chest radiograph and focused assessment with sonograph in trauma (FAST) were done which did not establish an immediate diagnosis. Computed tomography (CT) of the thorax revealed a tension pneumopericardium and moderate right hemopneumothorax, with multiple ribs fracture. An intercostal drainage tube (ICD) was inserted on right chest. The patient suffered a cardiac arrest and resuscitation measures were unsuccessful. The diagnostic pitfalls, the CT findings, possible clues to the diagnosis and the discussion of this rare case are presented in this case report.
\end{abstract}

Keywords: Tension Pneumopericardium; Cardiac Tamponade; Traumatic Pneumopericardium; Pneumo-Tamponade; Small Heart Sign

\section{Introduction}

Thoracic trauma is the second leading cause of death due to trauma after head injury. Patients with chest trauma who develop acute severe respiratory distress have a high mortality rate. If shock is also present in addition to respiratory distress, 75\% patients will die [1]. Tension pneumopericardium is defined as air in the pericardial sac compressing on the heart producing a tamponade effect. Most of the books mention about the fluid or blood causing tamponade but air in the pericardium causing tamponade is an extremely rare cause of cardiac tamponade which is not mentioned in most of the standard text books in emergency medicine and mentioned very rarely in the literature. Even without the development of tamponade, pneumopericardium has a mortality of 58\% [2]. Hence early diagnosis and rapid treatment is vital in restoring the life. Most importantly it is one of the reversible causes of cardiac arrest and prompt intervention can save life.

\section{Case Report}

A 65-year-old female patient was brought to our emer- gency department 25 minutes post trauma, with an alleged history of motor vehicle collision. Patient was unconscious since the time of incident. On arrival to the ED, patient was gasping, had gurgling sounds and a compromised airway. She was intubated immediately with manual cervical spine stabilization and ventilated. Blood pressure and peripheral pulses were not recordable, only the central pulses were present, heart rate was 86 beats per minute on the cardiac monitor which was showing sinus rhythm and Glasgow coma scale was 3/15. On primary assessment, patient was found to have distended neck veins, bony crepitus on right side of chest. No obvious external injuries were seen. Fluid resuscitation was initiated and a presumptive diagnosis of cardiac tamponade was made. Secondary assessment was unremarkable. We planned to perform pericardiocentesis and meanwhile bedside focussed assessment of sonography in trauma (FAST) was carried out, which was surprisingly negative for blood in the abdomen, pelvis and pericardial sac. Hence, pericardiocentesis was deferred in spite of cardiac tamponade being our most likely diagnosis. Bedside plain chest radiograph was done (Figure 1), which looked almost normal and could not give any clear diag- 
nosis.

The patient's systolic blood pressure increased to 80 $\mathrm{mm}$ of hg and peripheral pulse were weak even after aggressive fluid resuscitation with crystalloids and colloids, and the cause of hypotension was still not established. Patient was transfused with blood and a decision to get a computed tomography of the head, thorax and abdomen was made and patient was transferred to the CT room with on going resuscitation. The patient was transferred back to ED from the CT room with a diagnosis of tension pneumopericardium and moderate hemopneumothorax (Figure 2), with multiple rib fractures on right side. Thoracic surgeons were called for as this kind of case was being seen for the first time in our ED and there was no definite plan about how to approach the case. Immediately an intercostal drainage tube (ICD) was inserted on the right side of chest but during the procedure the patient went into cardiac arrest and the monitor showed asystole. Cardio pulmonary resuscitation (CPR) was started and cardiac resuscitation drugs were administered as per advanced cardiac life support (ACLS) guidelines.

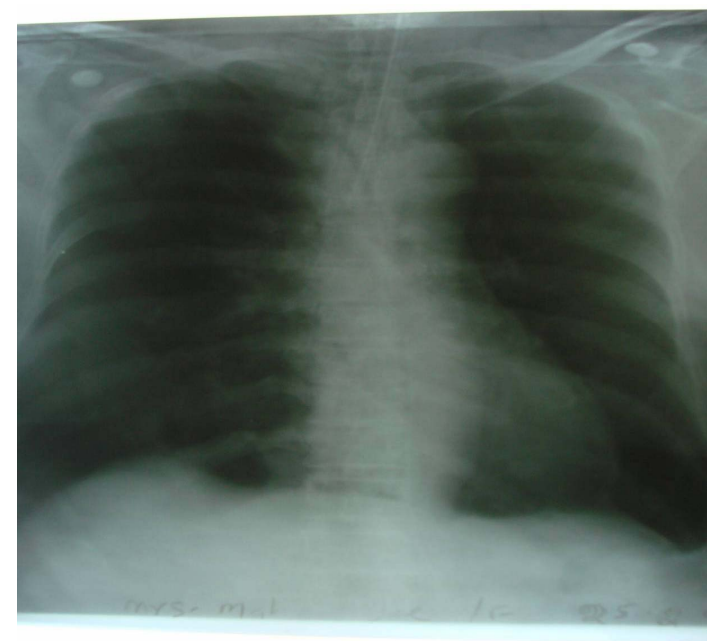

Figure 1. Bedside plain chest radiograph looked almost normal with no significant findings on it.

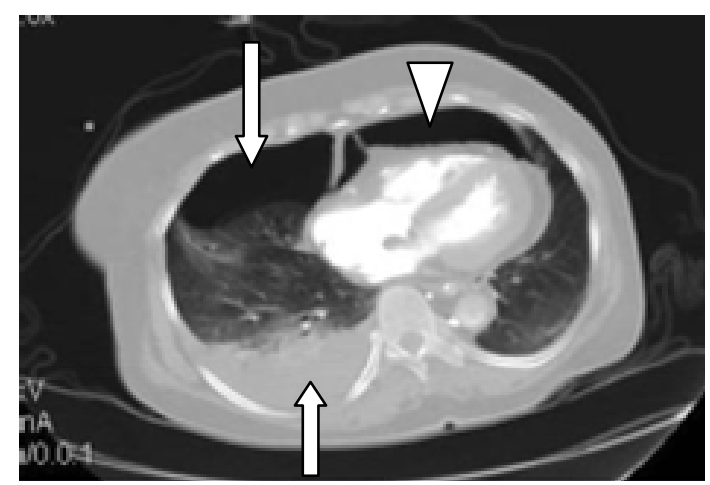

Figure 2. CT thorax showing right moderate hemopneumothorax (arrows) and pneumopericardium (arrow head).
Not sure whether this condition needs pericardiocentesis and also due to technical difficulties in accessing the pericardial sac in the presence of air, decision regarding pericardiocentesis could not be made. CPR was continued for 30 minutes but there was no return of spontaneous circulation (ROSC) during this period and the patient could not be revived hence was declared dead. The cardiac arrest was resistant to $\mathrm{CPR}$.

\section{Discussion}

Air within the pericardial sac is an unusual finding but, it has been recognized since 1844 (Bricheteau) [3]. Pneumopericardium following blunt thoracic trauma is an extremely rare case and pneumopericardium causing cardiac tamponade is even more infrequent condition with a very high mortality. It is not mentioned in most of the standard text books and literature. Most of the trauma courses teach about cardiac tamponade caused by blood but air causing tamponade is not addressed which is quiet possible entity. Presence of air in the pericardial sac does not always lead to compromise in the cardiac output but if present in excess, it might produce a tamponade effect making it a life threatening condition and needs emergency decompression by pericardiocentesis. Tension pneumopericardium produces the same physiological derangements as cardiac tamponade secondary to accumulated blood or other fluids and the presenting features are also similar. Beck's triad can be present in most cases. In most instances, pneumopericardium is always secondary to the dissection of air through the adjacent structures into the pericardial space. The air is trapped in the pericardium, which acts as a one-way valve, letting the air in but not out. This condition most often occurs in patients on mechanical ventilation, and in patients with blunt and penetrating chest trauma.

Probable mechanisms for pneumopericardium following blunt trauma are [4]:

- Direct tracheo-bronchial-pericardial communication;

- Pneumothorax with pleuropericardial tear;

- Penetration along pulmonary venous perivascular sheaths from ruptured alveoli to the pericardium.

Pneumopericardium is usually a self-limiting condition, requiring no specific treatment. Patients who are hemodynamically stable need close monitoring and those who are unstable, need immediate pericardiocentesis and drainage of air, followed by definitive surgical repair. In patients where there is a concomitant pneumothorax, inserting an intercostal drainage is usually sufficient, but in our case it was not adequate to save the life of our patient.

\section{Diagnostic Pit Falls}

- In most of the cases the diagnosis can be easily made on a plain chest radiograph, which shows the "small 
heart sign" in which the cardiothoracic ratio decreases as the heart is compressed and air can be seen surrounding the heart. As chest radiographs are taken in supine position in trauma patients, it makes diagnosing pneumopericardium an extremely difficult task.

- Air being a bad medium for ultrasonography, the diagnosis can be easily missed on FAST, which is performed routinely in the ED. When there is a strong suspicion of cardiac tamponade and in the absence of fluid in the pericardium, looking at the contractility of the heart can give a clue to the diagnosis of pneumopericardium.

- CT thorax is the best diagnostic test but it may not be possible to get a CT in all patients, especially if the patient is unstable and this diagnosis can be completely missed.

\section{Conclusions}

- Traumatic pneumopericardium is very rare, but can be seen in patients associated with high-speed blunt chest trauma.

- The diagnosis can be easily missed on FAST, and chest radiograph may not always be diagnostic, especially if it is taken in supine position.

- Computed tomography is the best test for diagnosing the presence of air in the pericardial space and may assist in establishing the diagnosis.

- Tension pneumopericardium requires immediate recognition and decompression to prevent cardiac tamponade which is fatal.
- Tension pneumopericardium is as serious as the tamponade resulting from hemopericardium and the clinical features are similar.

- Patients with mild to moderate pneumopericardium should be closely monitored, particularly those on positive pressure ventilation.

- Tension pneumopericardium is one of the life threatening injuries and should be kept in the differential diagnosis in any trauma patient presenting with circulatory collapse with no obvious cause.

- Tension pneumopericardium is a reversible cause of cardiac arrest.

\section{REFERENCES}

[1] T. G. Buchman, B. L. Hall, W. M. Bowling, G. D. Kelen, et al., Eds., "Thoracic Trauma," In: Judith Tintinalli's Emergency Medicine-A Comprehensive Study Guide, 6th Edition, McGraw-Hill, New York, 2004, p. 1595

[2] R. G. Cummings, R. L. R. Wesly, D. H. Adams and J. E. Lowe, "Pneumopericardium Resulting in Cardiac Tamponade,” The Annals of Thoracic Surgery, Vol. 37, No. 6, 1984, pp. 511-518.

[3] J. M. Douglas Jr., “The Pericardium,” In: Sabiston \& Spencer's Surgery of the Heart, 6th Edition, Saunders, Philadelphia, 1996, pp. 1378-2379

[4] D. H. Spodick, "Pericardial Diseases," In: Braunwald Zipes Libby's Heart Disease-A Textbook of Cardiovascular Medicine, 6th Editon, HIE Saunders, Philadelphia, 2001, p. 1832. 